\title{
A NON HEALING ULCER ON SCALP
}

\author{
Deepal J. Deshpande, Chitra S. Nayak, Sunil N. Mishra
}

Department of Dermatology, BYL \& TNMC Nair Medical College, Mumbai

Summary: Angiosarcoma is a soft tissue tumour with a dismal prognosis. We present a 74 year old male presenting with a non healing ulcer on the scalp. On histopathology a diagnosis of angiosarcoma was made. An early diagnosis and tumour size play a pivotal role in the survival of the patient.

Key words: Angiosarcoma; Non healing ulcer

\section{Introduction}

Angiosarcoma is an extremely malignant soft tissue sarcoma of endothelial origin. Because of its ambiguous presentation that usually mimics common benign dermatologic conditions, it is often diagnosed late. (1) Cutaneous angiosarcoma occurs in three settings: idiopathic angiosarcoma of the face, scalp and neck also called as Wilson-Jones angiosarcoma, angiosarcoma associated with chronic lymphoedema (Stewart-Treves syndrome) and post irradiation angiosarcoma. These sarcomas account for about $1 \%$ of all sarcomas and less than $0.1 \%$ of all head and neck cancers. Therefore, the rarity of angiosarcoma may impede early diagnosis. Angiosarcoma is usually found in elderly Caucasian men in their 70s. Angiosarcoma has the highest rate of lymph node metastases of all soft-tissue sarcomas of the head and neck, and distant metastasis may occur in up to $50 \%$, with the lung being the most common site. (1) Prognosis is dismal regardless of histologic type and therapy. Age, patient's sex, location, and clinical appearance have no effect on prognosis. However, tumour size has been found to correlate with favourable outcome, hence the need for early diagnosis and aggressive management. The aim of this case report is to highlight the importance of considering angiosarcoma in any elderly patient presenting with a non healing ulcer on the scalp.

\section{Case report}

A 74 year old male presented with a two month history of non healing ulcer with spontaneous bleeding on scalp and a violaceous bruise on the left eye. (Figure 1) The patient gave a history of applying some ayurvedic medications on the ulcer with no clinical response. There was no history of any local trauma or abscess. On examination there was a $10 \times 7 \mathrm{~cm}$ irregular ulcer with ill defined margins on the parietal area extending to the frontal and occipital areas of

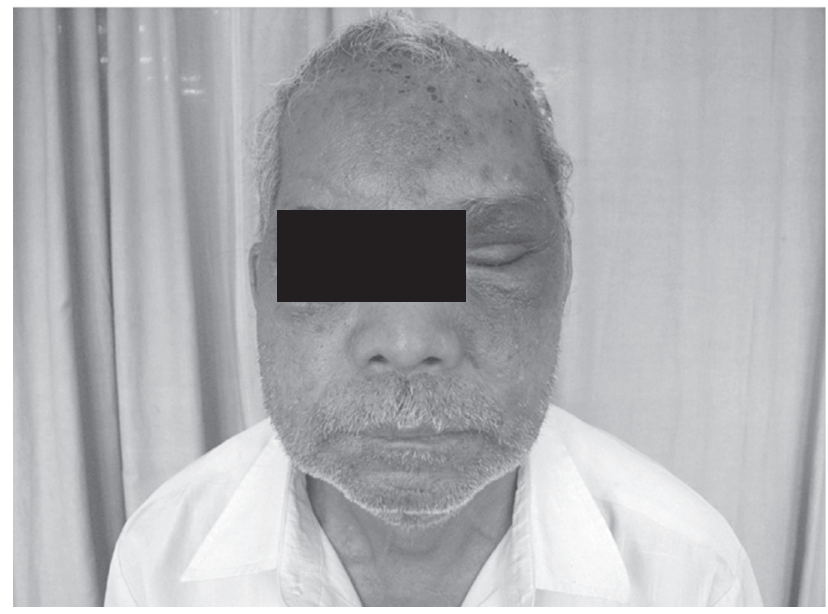

Fig. 1: Patient presenting with a bruise like lesion and edema of left side of face

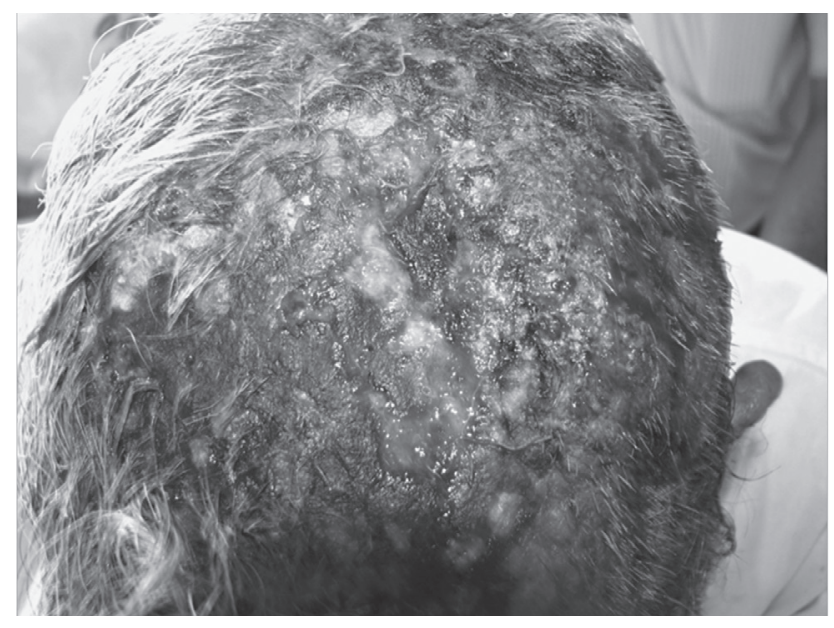

Fig. 2: Irregular ulcer with hemorrhagic crusting on scalp 


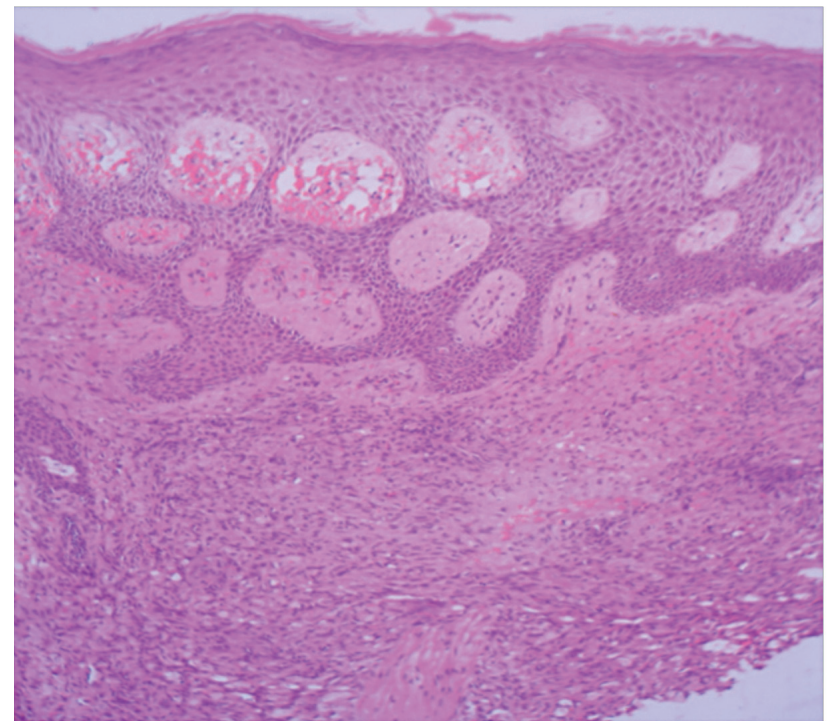

Fig. 3: On 10×, histopathology showing vascular channels infiltrating normal structures in a disorganized fashion

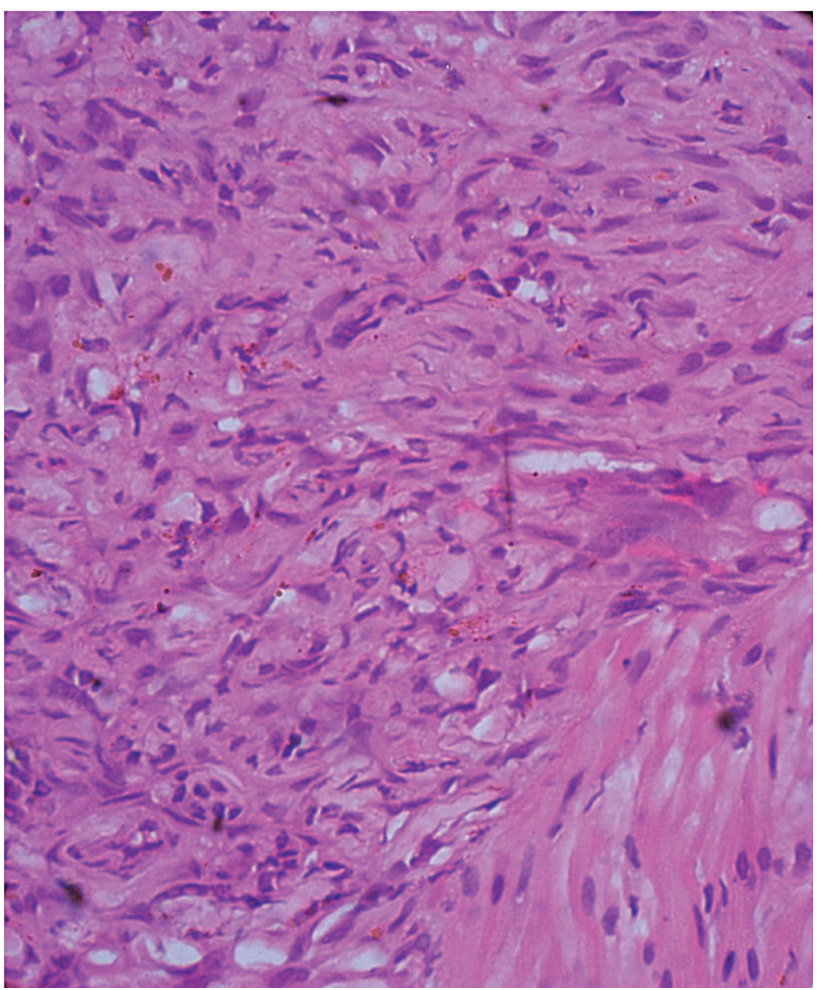

Fig. 4: On 40×, histopathology showing collagen characteristically lined by tumour cells in a pattern that has been described as 'dissection of collagen' the scalp. (Figure 2) The floor was covered with slough and hemorrhagic crusting. He had multiple, 1-3 cm diameter, discrete, stony hard submental and submandibular lymph nodes. A differential diagnosis of squamous cell carcinoma and cutaneous metastases was considered. All hematological and biochemical parameters were normal except for a low hemoglobin value of $10.2 \mathrm{~g} / \mathrm{dl}$. Biopsy from the edge of the ulcer was taken. (Figure 3) The histopathology (H\&E) showed a hyperplastic epidermis with increased vascularity. The collagen in the dermis was characteristically lined by tumour cells in a pattern that has been described as 'dissection of collagen'. (Figure 4) On X- ray chest there was blunting of the costophrenic angles bilaterally. A HRCT thorax revealed metastases in the chest. Based on the clinical, histopathological and radiological findings a final diagnosis of idiopathic angiosarcoma with pulmonary metastases was made. The patient finally succumbed due to spontaneous hemothorax caused by the pulmonary metastases.

\section{Discussion}

Angiosarcoma was first described by Livingston and Klemperer in 1926, when they reported a tumour of the scalp in a 38-year-old white male who later died from uncontrolled hemorrhage from the tumour. (2) Wilson Jones, 38 years after the first case was published (3), described a series of nine cases occurring on the face and scalp of elderly patients. These tumours are more commonly found in the elderly in the seventh decade with a male-to-female ratio of $2: 1$. (1) These tumours may be found in any location, although they have a predilection for the skin and superficial soft tissues in sharp contrast to most other sarcomas that are usually located deep within the body. Of those occurring on the skin and superficial soft tissue, over $50 \%$ are found in the head and neck region. In all types of angiosarcoma, the first sign may be an area of bruising, often thought by the patient to be traumatic. Dusky blue or red nodules develop and grow rapidly, and fresh, discrete nodules appear nearby. As the tumours grow, the oedema may increase and older lesions may ulcerate. Multifocality is a very frequent finding making surgical excision difficult especially on the face and scalp. Dissemination occurs early, with the first visceral deposits usually being in the lung and pleural cavity. On histopathology, vascular channels infiltrate the normal structures in a disorganized fashion lining available tissue space with a layer of endothelial cells. The collagen is characteristically lined by tumour cells in a pattern that has been described as 'dissection of collagen'. Less well differentiated tumours show more atypical, pleomorphic, endothelial cells, often with a spindle-cell morphology and loss of vascular pattern with proliferation of cell masses. (4) Immunohistochemical studies have indicated that antibodies to CD31 are the most reliable markers for routine use, compared with antibodies against von Willebrand factor and CD34. Podoplanin antibodies are used to differentiate a subset if angiosarcoma showing both blood 
vessel and lymphatic lineages. (5) The treatment includes surgical excision with wide margins, radiation therapy, immunotherapy, and chemotherapy. A combination of any of these forms of treatment can be used with some success. (6) The single most important prognostic factor is the size of the lesion at the time of presentation, making early diagnosis crucial.

\section{Conclusion}

Angiosarcoma carries an inevitable mortality if not diagnosed promptly. No single treatment modality is efficacious if the diagnosis is delayed. A tumour size of less than $5 \mathrm{~cm}$ is associated with a better prognosis, making early detection important. When feasible, wide surgical excision is advocated with subsequent radiation therapy. Because of the tumour's propensity for local recurrence and distant metastases, even after a prolonged disease-free state, life-long surveillance, especially pulmonary surveillance, is strongly advised.

\section{References}

1. Jones EW. Malignant angioendothelioma of the skin. Br J Dermatol 1964; 76: 21-39.

2. Livingston SF, Kemperer P. Malignant angiomas; with reference to question of sarcoma due to roentgen ray. Arch Pathol Lab Med 1926; 1: 899-910.

3. Liu AC, Kapp DS, Egbert B, Waters L, Rosen JM. Angiosarcoma of the face and scalp. Ann Plast Surg 1990; 24: 68-74.

4. Brightman LA, Demierre MF, Byers HR. Macrophage-rich epithelioid angiosarcoma mimicking malignant melanoma. J Cutan Pathol 2006; 33: 38-42.

5. Kahn HJ, Bailey D, Marks A. Monoclonal Antibody D2-40, a New Marker of Lymphatic Endothelium, Reacts with Kaposi's Sarcoma and a Subset of Angiosarcomas. Mod Pathol 2002 Apr; 15(4): 434-40.

6. Pawlik TM, Paulino AF, McGinn C, Baker LH, Cohen DS, Morris JS, et al. Cutaneous angiosarcoma of the scalp: a multidisciplinary approach. Cancer 2003; 98(8): 1716-26.

Received: 06/04/2011 Accepted in revised form: 24/10/2011

\section{Corresponding author:}

Chitra Nayak, Professor \& head BYL \& TNMC Nair Hospital, Mumbai Central, Mumbai, India; e-mail: chitra1202@yahoo.co.in 